\title{
TABLEAUX GRAMMATICAUX.
}



La langue turque est une ramification d'une grande souche de langues, qui probablement tira son origine de l'Altaï et des montagnes qui s'y rattachent. Cette souche donna le jour à quatre grandes ramifications ou familles de langues: le Turc, le Finnois, la langue Mongole et celle des Tongous. *)

Le Turc est parlé dans une grande partie de l'Asie centrale et occidentale.

Cette langue se divise en trois dialectes principaux:

1. le Tchagataïen ou dialecte oriental qu'on parle dans le 'Turkistan. Des livres rédigés dans ce dialecte ont été imprimés à Kazan seulement.

2. le Tarfare proprement dit dans le Kyptchak et dans la Sibérie.

3. le dialecte occidental, c'est -à - dire l'osmanli, dans le SudEst de l'Europe et l'Asie mineure. **)

De ces dialectes le premier et le dernier ont acquis une importance historique, et ont exercé la plus grande influence sur le développement de la civilisation Mahométane.

Dans la plupart des dialectes turcs il est entré un grand nombre de mots arabes et persans, ce qu'il faut attribuer à linfluence des littératures arabe et persane.

Les lettres arabes que les Turcs mahométans ont adoptées pour écrire leur langue sont bien peu propres à en représenter les sons; cette observation s'applique surtout aux voyelles.

Voici l'alfabet ottoman avec la prononciation.

*) cf. Schott $\ddot{C}$ ber das altaische oder Finnisch-tartarische Sprachengeschlecht. 1849.

$\left.{ }^{* *}\right)$ cmp. Berésin: système des dialectes turcs. Kazan 1849. pag. 26, 27. il faut $y$ ajonter 4. le dialecte des Yakoutes peuple de la Sibérie orientale, et 5. celni des 'Tchouvaches parlé dans le gouvernement de Kazan. 
II

\begin{tabular}{|c|c|c|c|c|c|c|}
\hline NOMS. & 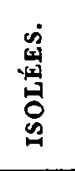 & 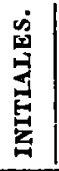 & 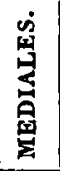 & 我 & $\begin{array}{c}\text { VALEUR DES LETTRES } \\
\text { EN } \\
\text { CARACTËRES FRANCAIS. }\end{array}$ & 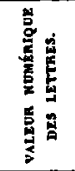 \\
\hline Elif & 1 & & & $l$ & leger hiatus & 1 \\
\hline $\boldsymbol{B} \boldsymbol{d}$ & ب & ! & + & مب & $B$ & 2 \\
\hline$p_{d}$ & ب ب & ه. & 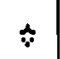 & مب & $p$ & \\
\hline$T \grave{e}$ & $\underset{8}{8}\{$ & ت & $\ddot{a}$ & $\ddot{\ddot{x}}$ & $T$ doux & 400 \\
\hline $\mathcal{S} \dot{e}$ & ث & $\dot{s}$ & $\dot{\alpha}$ & نه & $S$ et $G$ & 500 \\
\hline$D j i m$ & $\tau$ & $ج$ & Tج & 尺 & $D_{j}$ & 3 \\
\hline Tchîm & z & 7 & $\div$ & $\widehat{c}$ & $T_{c h}$ & \\
\hline $\boldsymbol{H a}$ & $\tau$ & $>$ & $>$ & $\varepsilon$ & $H$ aspiré & 8 \\
\hline Khy & $\dot{z}$ & $\dot{>}$ & $\doteqdot$ & $\dot{\mathrm{z}}$ & $K h$ & 600 \\
\hline Dal & s & & & $u$ & $D$ & 4 \\
\hline Zal & $j$ & & & $\dot{i}$ & $z$ & 700 \\
\hline $\boldsymbol{R} \boldsymbol{e}$ & , & & & F & $R$ & $2 \mathrm{co}$ \\
\hline $\boldsymbol{Z} \grave{e}$ & j & & & j & $z$ doux & 7 \\
\hline$J \grave{e}$ & $\dot{j}$ & & & $\dot{s}$ & $J$ & \\
\hline $\sin$ & un & w & $m$ & m & $s$ & 60 \\
\hline Chin & ش & $\dot{H}$ & $\ddot{~}$ & ش & $C h$ & 300 \\
\hline Sad & $\infty$ & מ & $a$ & v & $\mathcal{G}$ dur & 90 \\
\hline Zad & 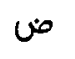 & $\dot{\infty}$ & ص & ض & $Z$ dur & 800 \\
\hline Thy & $b$ & $b$ & $b$ & $b$ & $T h$ dur & 9 \\
\hline Zhy & ظذ & $b$ & $\dot{b}$ & b & $Z$ dur & 900 \\
\hline $\operatorname{Ain}$ & $\varepsilon$ & $=$ & $*$ & $\varepsilon$ & un hiatus assez sensible & 70 \\
\hline Ghain & $\dot{\varepsilon}$ & $\dot{\varepsilon}$ & $\dot{\boldsymbol{k}}$ & $\dot{\varepsilon}$ & $G h$ & 1000 \\
\hline $\boldsymbol{F} e^{\prime}$ & ف & $\dot{j}$ & $\dot{2}$ & ف & $\boldsymbol{F}$ & 80 \\
\hline Qaf & ق & $\ddot{3}$ & $\ddot{\mathbf{z}}$ & $\ddot{\theta}$ & $Q \mathrm{dur}$ & 100 \\
\hline Kef et Guef & $ك$ & $s$ & $\alpha$ & 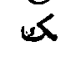 & $K$ et $K i, G$ & 20 \\
\hline Saghyr Nun & $\ddot{3}$ & $\dot{s}$ & $\dot{\boldsymbol{x}}$ & $\ddot{\leftrightarrow}$ & $N$ nasal & \\
\hline
\end{tabular}




\begin{tabular}{|c|c|c|c|c|c|c|}
\hline NOMS. & 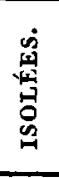 & 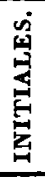 & 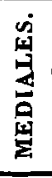 & 窝 & $\begin{array}{c}\text { VALEUR DES LETTRES } \\
\text { DE } \\
\text { CARACTỲHES FRANCAIS. }\end{array}$ & 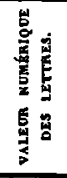 \\
\hline$\overline{\text { Lam }}$ & $J$ & 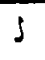 & 1 & $\lambda$ & $\bar{L}$ & 30 \\
\hline Mim & $p$ & ه & + & $r$ & $M$ & 40 \\
\hline Nun & $\dot{0}$ & $\dot{j}$ & $\dot{i}$ & ن & $N$ & 50 \\
\hline$V a v$ & , & & & g & $V$ & 6 \\
\hline$H \grave{e}$ & 8 & $\approx$ & $t$ & $\alpha$ & $\begin{array}{l}\boldsymbol{H} \\
. .\end{array}$ & 5 \\
\hline $\boldsymbol{J}$ & $\checkmark$ & بـ & 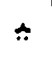 & $\checkmark$ & $\ddot{J}$ & 10 \\
\hline
\end{tabular}

\section{REMARQUES SUR LES LETTRES.}

\section{Consonnes.}

1. Les consonnes $\uplus, \infty, ظ$ ne se rencontrent que dans les mots arabes.

2. Le $\dot{\zeta}$ ne se trouve qu'au milieu ou à la fin d'un mot; il se prononce comme $n$ nasal et quelquefois comme $m$, mais originairement il avait la valeur de l'articulation $n_{G}$ en allemand ou en anglais. Quoiqu'on ne distingue pas dans les manuscrits turcs 6 de ك on le fait très souvent dans les impressions occidentales pour faciliter l'intelligence du texte.

3. Il n'y a pas de mots Turcs qui commencent par les lettres, ou $\dot{\varepsilon}$.

4. Le غ̇ghain entre deux voyelles est très adouci; il a quelquefois la valeur d'un $v$ ou d'un $h$ p. e. آغ avyz, آغاج ahadj. Mais à la fin d'une syllabe on le prononce comme ou p. e. طاغ thaou. ك se prononce entre deux voyelles et à la fin d'un mot comme $\ddot{i}$.

5. Les mots de plusieurs syllabes finissant par $\ddot{\jmath}, \omega(k), *$ changent ces lettres en $\dot{\varepsilon}, \circlearrowleft(g), \supset$ quand ils prennent une terminaison grammaticale. 
6. Au commencement des mots on prononce souvent $\downarrow$ comme .

7. L'accent tombe généralement sur la syllabe finale; le mot peut changer de signification suivant la place de l'accent p. e. إبر ë̈er selle St éier si.

On évite le hiatus en insérant un $v$ p.e. قَبِيو qapouia ou en reprenant un $n$ essentiel qui aurait été supprimé après les consonnes seules p.e. كلينك kedinin.

\section{VOYELLES.}

1. Les voyelles turques se divisent en deux classes:

a. les voyelles fortes $a, o, o u, y$.

$b$. les voyelles faibles $e, e u, u, i$.

Une voyelle est forte ou par sa nature, ou par l'influence d'une voyelle forte qui la précède.

2. Pour exprimer toutes ces voyelles on fait usage des consonnes arabes $l, g, \mathcal{G}$, s et quelquefois mais bien rarement du p.e. عورت auret. On les remplace aussi par les signes des voyelles arabes correspondantes: - pour $a, e$; - pour $y, i$; pour o, ou, eu, $u$; dont le redoublement exige la prononciation nasale: $\stackrel{s}{\longrightarrow}$ pour

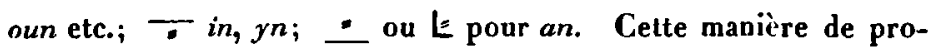
noncer les voyelles est employée de préférence dans les mots arabes.

3. Ordinairement on se content d'écrire une seule voyelle et il y a beaucoup de mots où l'on n'en met point du tout. C'est pour cela que nous ajoutons dans les dictionnaires turcs la prononciation des mots. Les textes tchagataïens seuls nous présentent toutes les voyelles des mots turcs.

Rem. On omet de préférence la voyelle dans les syllabes fermées. Dans les syllabes ouvertes on l'exprime toujours quand elle est la seule lettre radicale ou quand elle termine le mot p. e. بلمكك

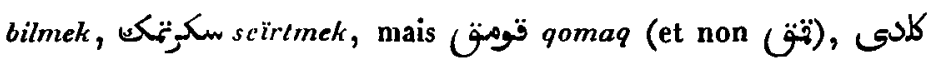


gueldi (et non كلى). La voyelle ne peut être supprimée au commencement d'un mot.

3. Les consonnes $\tau, \dot{\tau}, \varepsilon, \dot{\varepsilon}, \ddot{\xi}, \omega, b$ sont toujours accompagnées de voyelles fortes, soit que la voyelle les suive ou qu'elle les précìde.

Rem. 1. Les consonnes 5 se prononcent dans les mots turcs avec des voyelles faibles. Quand l'ćlif signe d'un $a$ fort précède ou suit une de ces consonnes, le mot est d'origine étrangère p. e. كاتب kiatib. Si le كst suivi d'un elif ou d'un, on prononce ces voyelles en insérant un $i$ trìs bref p.e. كون guiun.

Rem. 2. Comme il n'y a pas de différence de prononciation entre $\omega$ et $\omega, b$ et $\boldsymbol{w}$ on choisit $\omega$ et $b$ si le $\boldsymbol{S}$ ou le $\boldsymbol{T}$ doit ctre prononcé avec une voyelle forte. Cependant on trouve aussi i et wavec des voyelles de cette classe p.e. تو نو

4. Le I dans les mots turcs précédé d'une consonne se prononce

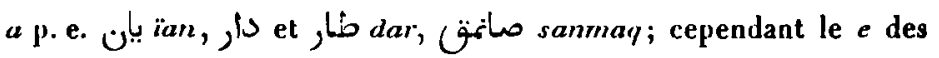
terminaisons grammaticales s'écrit quelquefois i p. e. كلار guelen ce qui vient. Au commencement des mots le I peut exprimer $a, e, y, i$

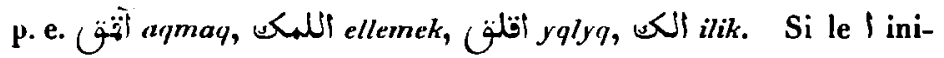
tial a la valeur de a nous l'écrivons avec un medda $\%$.

Rem. Il faut observer ici que dans les mots arabes et persans le $l$, et $\mathbf{v}$ représentent des voyelles longues, ce qui n'a jamais lieu dans les mots turcs.

5. Le, voyelle représente les voyelles dures o, ou et les voyelles molles $e u, u$. Si des consounes dures se rencontrent dans un mot, le, ne peut avoir d'autre valeur que celle d'un o ou d'un ou; p. e. قول qoul, قيون qoun. Accompagné de consonnes molles le, se prète au son de o, ou, eu, u, mais $s$ et $\dot{S}$ se pro-

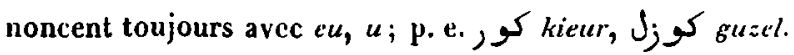


Si la syllabe précédente a pour voyelle un $e$ le, a la valeur de $i$; si elle a pour voyelle un a le vav se prononce comme $y$; p. e. آيو aiz.

Le, représente aussi oou, ov, euv, av, ev; p. e. صور soouq froid, تومتز mieux قومون goumaq chasser, كونه gueuvdé corps,

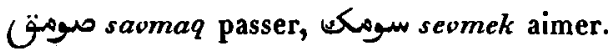

Rem. Quelques mots écrits de la même manière se distinguent par une prononciation différente du g; p. e. قول qoul esclave, قو qol bras.

6 . Le $\checkmark$ voyelle correspond aux voyelles $i, y$ et $u$; plus rarement à, ou et aux diphtongues $a \ddot{a}$, ë̈, oui. Le $\checkmark$ voyelle radicale n'est pas autre chose que $i, y$ ou diphtongue; cf. ميرى miri trésor public, كيشث qych hiver, كيرمت qurmaq rompre, سوزى seuzu sa

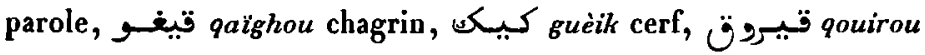
queue.

7. Le Hè \& voyelle a la valeur de $a$ ou de $e$; on ne le trouve qu'à la fin des syllabes et des mots; p. e. هر د deré vallée, قره qara noir. Comme voyelle il ne se lie jamais à la consonne suivante;

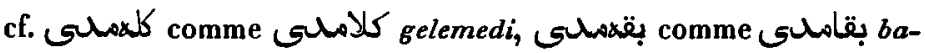

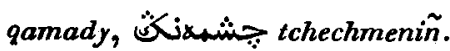

8. Un Elif au commencement des mots est souvent suivi d'un , ou d'un 5 ; il résulte de cette combinaison une diphtongue ou quelque chose d'analogue; p. e. I av chasse, glev maison, $\mathcal{J}^{\top} a_{i}$

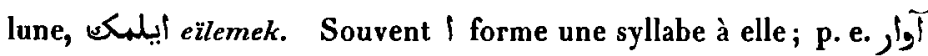
avar injustice, آئز azaq pied, أيو eii bon. Mais si un mot turc commence par une voyelle, dont le représentant est, ou $\boldsymbol{\xi}$ on lui ajoute comme appui un $\mid$ qui précède; p. e.

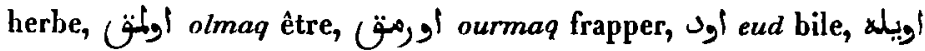

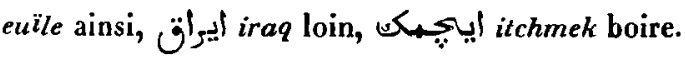


9. Tous es signes de rapports grammaticaux ont pour voyelles $a, e$ ou $y, i ; a, e$ est la voyelle du datif et de quelques terminaisons du verb:. Les autres cas du nom, les pronoms suffxes et la plupart des afixes du verbe ont $y, i$. Le choix entre les voyelles $a, y$ ou $e, i$ ie décide suivant que la voyelle est forte ou faible.

$i$ ne: peut être placé qu'après $e$ ou $i$; p. e. Jl el,

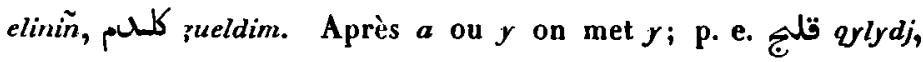
أيان äaghy, أباغى aldy.

Après ou et $u$ on change $i$ en $u$ quoiqu'on écrive $v$; p. e. سوز , أوز ,سوزم ,سوز sumu, كيلى guldu-mu?

Iprès o et ou l'i devient ou; p. e. قول ولن qol, qolouñ,

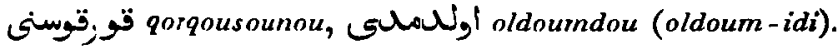

10. Le premier $i$ changé exerce son influence sur toutes les voyelles suivantes, mème si le mot devient très long. Mais quelques petits nots ajoutés s'opposent à cette influence. Cela a principalement lieu dans كى $\mathbf{~}$ dont le $k$ résiste à linfluence d'une vo-

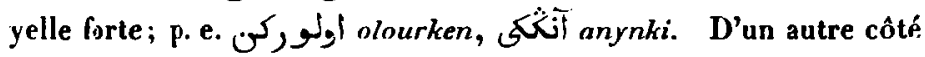
la syllabe qui désigne le temps présent sonne toujours io et exige une vcyelle forte aprìs elle quand mème une voyelle faible précéderait p. e. ديور deioroum.

L'influence d'une voyelle sur la suivante est annulće si une voyell: d'une autre espèce se glisse entre les deux; p. e. كوربدم gurmelim, ublol olmadyrn.

11. La voyelle du participe en $r$, au moyen duquel on forme le présert et l'imparfait, est généralement $a, e$. Souvent elle est $i$, mais cet $i$ peut devenir $y, u$, ou pour se conformer à la voyelle ra-

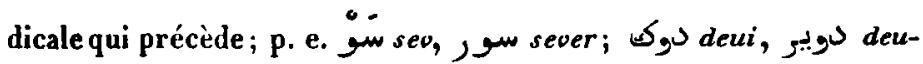

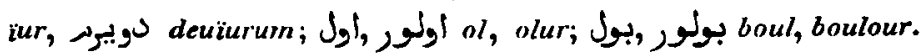


VIII

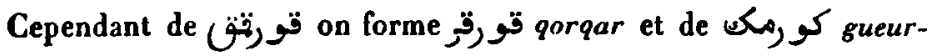
mek, كور gueurer.

12. Quelques mots de plusieurs syllabes ne se conforment pas aux lois de l'harmonie des voyelles; p. e. Ul elma pomme, avret femme. Dans les mots arabes cela a lieu plus souvent; p. e. قلم qalem plume, adem homme, زآ مان zeman temps, مبارك mubarek béni, مخنَ muzakker masculin.

\section{LES SIGNES ORTHOGRAPHIQUES.}

1. Djezm * indique que la consonne, au-dessus de laquelle il est placé, ne doit être accompagnée d'aucune voyelle dans la pro-

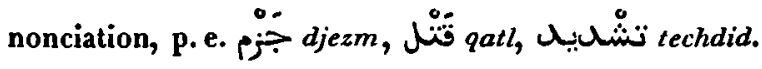

2. Techdid * se place sur une consonne quelconque pour indiquer qu'elle doit être redoublée, p. e. Uمَّ mohammed, قوّة quvoet.

3. Medd - ne se met pour l'ordinaire que sur l'élif, il indique dans les mots arabes que le son de cet Elif doit être prolongé, p. e. T akhyr, ${ }^{\top}$ T an. Au commencement des mots turcs le Medd désigne la prononciation a, p. e. آك آكلامن añamaq.

4. Le Hamz se place:

1) dans les mots arabes au-dessus de tout Elif consonne, p. e. ثأثير te'écir, تأثيب te'ebid;

2) le , et le $\mathbf{v}$ sont munis de ce signe lorsqu'ils remplacent

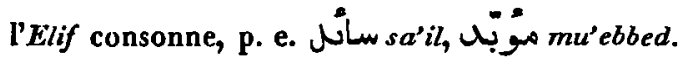

3) le Hamz seul sans aucune lettre pour le supporter à la fin des mots tient lieu d'un 1, , ou $\checkmark$ consonne qui est

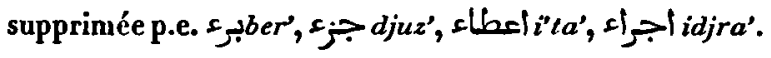

4) Après une voyelle finale exprimée par r ou $\mathbf{G}$ le Hamz remplace la voyelle $i$ qui est alors marque d'un génitif exprimé à la persane. Elle peut désigner en outre le 
rapport entre un substantif et son adjeptif si ce dernier est postposé. Enfin il se place quelquefois au lieu de بي (Acc.) à la fin des mots terminés par une voyelle.

5. Le Vasle $\bullet$ se met sur l'Elif de l'article arabe Ji pour indiquer que ce I se doit prononcer avec la voyelle du mot précédant.

\section{LE NOM.}

Nous donnons d'abord les paradigmes pour montrer les lois de l'harmonie des voyelles (de l'euphonie) appliquées à la déclinaison des nots turcs.

I. Noms finissants par une consonne.

\begin{tabular}{|c|c|}
\hline $\begin{array}{l}\text { Singulier. } \\
\text { Jl el main }\end{array}$ & Ju dil langue \\
\hline ڤ elin & Sل diliñ \\
\hline دil elé & دن dilé \\
\hline 나 eli & ل \\
\hline J I ب el el & يا دي \\
\hline
\end{tabular}

N. ثاث qack sourcil ulli elden Pluriel.

N. قانشلر qachlar اللر

G. قاثشلركث qachlaryn ellerin

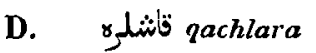
ellere:

A. قاثثلرى qachlary illeri

V. يا قالثنلر ia qachlar يا اللر

Abl. قانثلرد qachlardan ellerden Singulier.

N. قو goul esclave qol bras uls dilden

G. قولكث و qouloun وقولك وoloun is diller comme !)

D. قولي

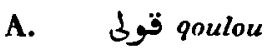
و قولم سوز seuz mot

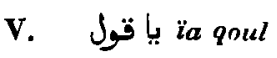
قو سوز سوز سوزي seuzé

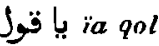
سوزى seuzu Abl. قولى qouldan و qولى

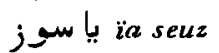
ئ seuzden 
Pluriel.
N. قوللر qoullar
qollar
سوزلر seuzler
G. etc.
comme
comme
قانثلر
الر

II. Noms finissants par 3 et 3 .

$$
\text { Singulier. }
$$

N. قو qualyq poisson

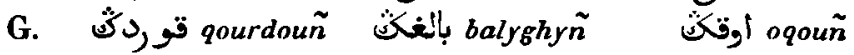

D. بالغ balygha

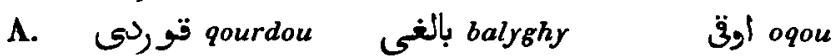

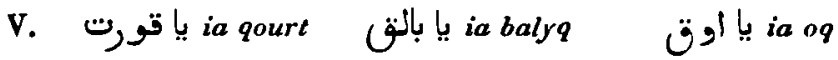

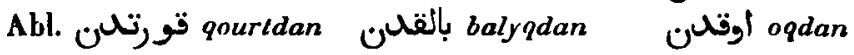

Pluriel.

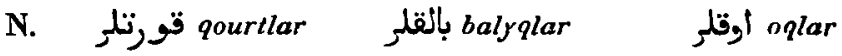

III. Noms finissants par des voyelles.

$$
\text { Singulier. }
$$

N. بابا baba père

قيو qapy porte

जur kedi chat

G. بابانك babanyñ

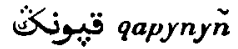

كابنغ kedinin

D. بابايx babaia

qapyia

كلئي كediie

A. بإبايى babaiy

rapyiy

كtedii

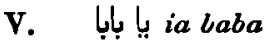

ia qapy

ئ ئ ia kedi

Abl. بابان babadan

qapydan

كبيلن kediden

Pluriel.

N. بابالر babalar

وapylar

كediler

$$
\text { Singulier. }
$$

(irrégulier)

Pluriel.

N. صو sou eau

A. صوبيى souiou

G. صوبك souioun

V. يإصو صو

D. souia

Abl. صون soudan

N. صول soular 


$$
\text { R e m ar qu ues. }
$$

1. On voit d'abord que les cas des mots se forment par l'addition de différentes lettres: un is marque le génitif, un $>$ le datif, un $ى$ l'accusatif et la syllabe $ن$ l'ablatif.

On y peut ajouter encore un cas que l'on peut appeller le commoratif ou locatif parcequ'il a la valeur de "rester, demeurer dans" et que l'on forme en ajoutant la syllabe 8 , p. e. sull eldé dans la main, سويله seuzdé dans le mot. La mème forınation a lieu dans le pluriel suر ellerdé dans les mains, قولرده qollarda dans les bras etc.

2. Les voyelles au moyen desquelles on joint ces lettres de cas ì un mot quelconque, sont choisies selon les règles de l'euphonie que nous avons expliquées p. VII.

3. Le $\ddot{\mho}$ et le ${ }^{-}$se changent en $\dot{\varepsilon}$ et $\supset$ si le mot reçoit des additions grammaticales, cf. p. III, 5. Cette règle cependant n'est pas applicable à un nombre de mots monosyllabes, tels que بوق أو

4. Les mots fimissants par une voyelle reprennent au génitif le Noun radical de ce cas. Dans le datif et l'accusatif des mots de ce genre on évite le hiatus en insérant un $\mathbf{G}$ (cf. p. VI, 6.).

5. Le pluriel turc est formé par la syllabe \ prononcée lar, si la voyelle radicale est forte et ler, si la voyelle radicale est molle. Le pluriel des noms qui ne sont pas d'origine turque se forme en

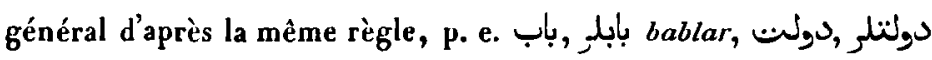
devletler, $\mathrm{J}$, $\mathrm{Guller.}$ Mais il faut savoir que nous trouvons assez souvent le pluriel original des noms persans. On en a deux, l'un pour les mots qui signifient un être humain, on le forme en ajoutant la syllabe $\mathfrak{ن}^{\prime}$ an, et un autre pour les noms persans qui signifient un objet inanimé, on le forme en ajoutant la syllabe Lo; 


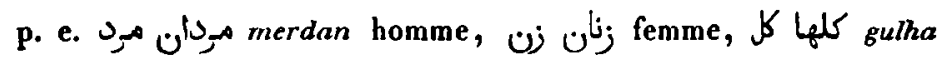

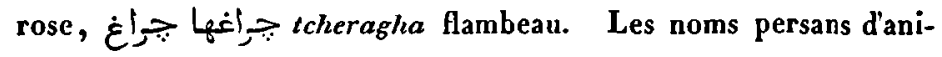

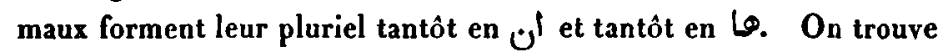
même des noms d'objets inanimés munis de la syllabe $u^{\prime}$ pour le pluriel. Si les noms persans qui devaient former leur pluriel en I finissent par un 8 ils forment leur pluriel au moyen d'un $\int_{\boldsymbol{g}} \mathrm{cf}$.

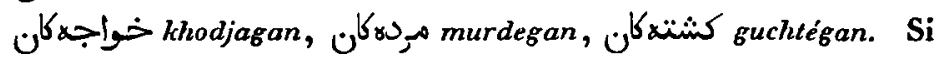
les mots de ce genre devaient former leur pluriel en 10 ils perdent généralement le r devant cette syllabe, خانx khanha de خانها

Les mots arabes sont susceptibles de trois nombres grammaticaux, le singulier, le duel et le pluriel; le duel se forme en $\mathcal{u}^{l}$ an pour le nominatif et en يin pour le génitif et l'accusatif; cf.

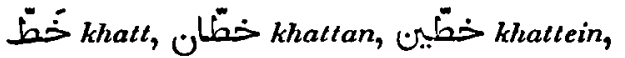

$$
\begin{aligned}
& \text { دولتنبن ,دولتان , دولني }
\end{aligned}
$$

Le pluriel régulier arabe des noms de forme masculine est en 'oun pour le nominatif et en $\mathcal{U}^{-}$- in pour le génitif et l'accu-

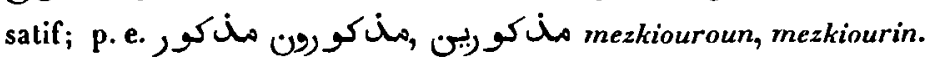

Le nom féminin arabe fonissant en $\ddot{8}$ ou forme le pluriel ré-

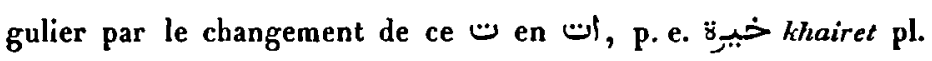
خيرات khairat. Il y a un grand nombre de formes de pluriels irréguliers pour les mots arabes. Ces pluriels irréguliers qui causent beaucoup d'embarras aux éludiants sont rangés dans notre glossaire parmi les autres mots selon l'ordre alphabétique.

6. Les Turcs ne distinguent jamais le genre par des terminaisons. Quelquefois ils emploient des mots signifiant homme ou femme, mâle ou femelle en les plaçant devant le mot. Ces mots

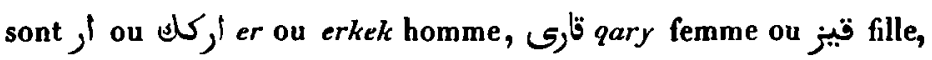

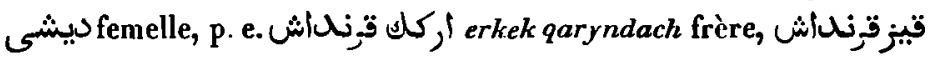

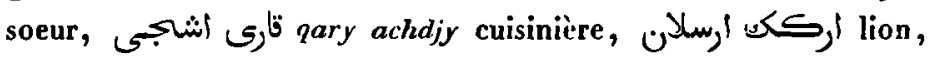
dichi arslan lionne. 
i. Les mots arabes de la forme لفe af'al qui a généralement la valeur du comparatif, forment leur féminin en فُ fưla. Cette

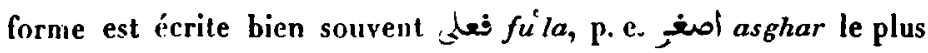

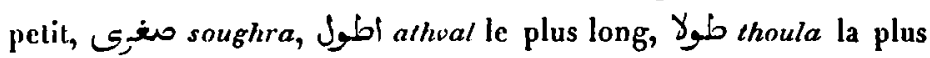
longue.

8. Le diminutif des mots tures est formé par l'addition de la

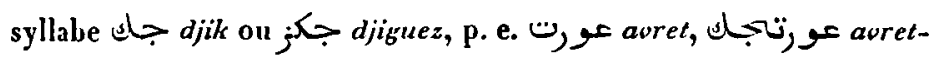
djik, avreldjiguez une petite femme.

9. Les pronoms possessifs sont ajoutés comme suffixes aux noms. Nous en donnons les paradigmes.

$$
\text { Singulier. }
$$

قانثم

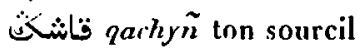
gachymyz notre sourcil qachyñyz votre sourcil قachy son sourcil qoloum mon bras وoloun ton bras

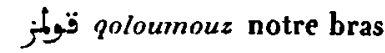
و qolouñouz votre bras قو qolnu son bras.

\section{Pluriel.}

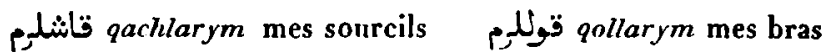
qollaryn tes bras

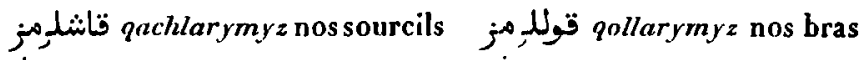
qollaryñyz vos bras

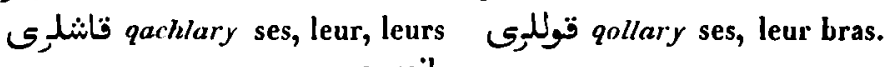
sourcils

\section{Singulier.}

$$
\text { مxلvl, validem ma mère }
$$$$
\text { ثँ vall, valideñ ta mère }
$$

زoxull, validemiz notre mère گेoull, valideñiz votre mère जurull, validesi sa mère
Sl elim ma main ¿) elin المز elimiz eliniz

(ل) eli 
Pluriel.

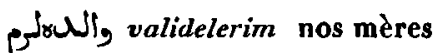
گेunlt, validelerin tes mères ivoll, validelerimiz nos mères Şuلl, valideleriniz vos mères ज列 valideleri ses, leur, leurs اللبر ellerm mes mains ثاث ellerñ البلرمن البر ellerimiz اللمرثز mères

Singulier.

Pluriel.

سوزم seuzum ma parole سوزگ seuzuñ سوزلم seuzlerim nes paroles

سوزئ seuzumuz سوزلمرگ seuzlerin

سوز seuzuñus seuzlerimiz

سوزى seuzu سوز سوخز سوزلز سوكن seuzleri

Noms suivis de l'affixe de la troisième personne du singulier.

a) terminé par une consonne.

b) terminé par une voyelle.

Singulier.

قولى qoulou son esclave

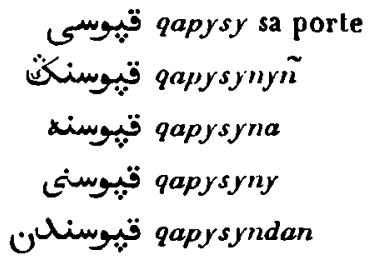
ؤنulounouñ qoulouna ؤنulounou qouloundan Pluriel.

qoullary ses esclaves قيبولزى gapylary ses portes qoullarynyñ qoullaryna *) قوللرينى qoullaryny qapylarynyn qapylaryna *) قيولرِينى qapylaryny. qoullaryndan وapylaryndan

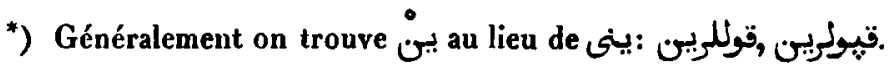




\section{L'AIJIECTIF.}

1. L'arljectif turc qui sert à qualifier les noms, les verbes et d'autres adjectifs, suit toutes les rigles du substantif qu'il remplace biell souvent. Il ne subit jamais aucun changement car il reste le mème pour tous les genres, nombres et degrés de comparaison; cf. أبيو أبر قارى eiii qary bonne femme pl.

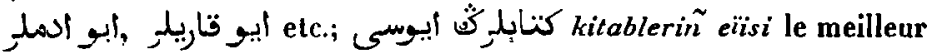
des livres.

2. Pour renforcer la valeur de l'adjectif on emploie quatre mots : S ou L L dakhy ou daha davantage, encore; ce mot a alors le sens de comparatif, cf. قورت اتتلن دها غيرتلو دourt itden daha ghairenli dir le loup est plus courageux que le chien. ثै eñ au su-

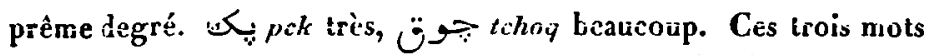
donnertt à l'adjectif le sens du superlatif, ألملرك اكث أيوسى ademleriñ ez̃ eïsi le meilleur des hommes.

3. La syllabe $\ddot{\xi}$, raq ou $\sigma, r c k$ ajoutéc aux adjectifs forma anciennement une espèce de comparatif, p. e. يشاك iechil, يشلرك iechilrek plus vert, $\underset{\mathcal{G}}{ }$ Jl allchaq bas, altchaqraq plus bas.

4. Le comparatif et le superlatif des adjectifs persans se forment

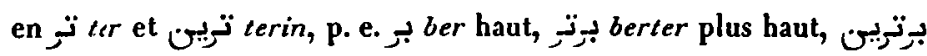
berterin le plus haut.

5. Les adjectifs d'origine arabe suivent parfois les mêmes règles que les noms arabes; ils forment généralement leur féminin par l'addition de 8, p.e. un due et un pluriel. Pour les degrés de comparaison il sera utile d'en savoir quatre formes: فاعلى fa'il simple adjectif, p. e. Sle a'lim un homme qui sait; عليبم fa'ìl, p. e. عبل un homme qui a l'habi-

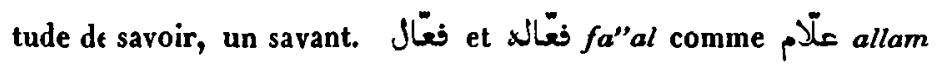




\section{XVI}

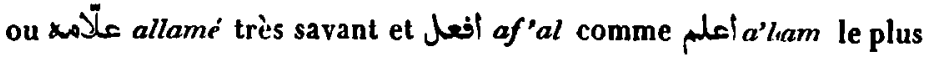
savant. (cmp. pag. XIII, 7.)

\section{LES NOMS DE NOMBRE}

s'emploient sans subir aucun changement à moins qz'ils ne soient pris substantivement.

Les noms de nombres cardinaux simples de la largue ottomane sont les suivants:

$$
\begin{aligned}
& \text { bir un } \\
& \text { otouz trente } \\
& \text { أببكى iki deux } \\
& \text { z ol utch trois } \\
& \text { تر qyrq quarante } \\
& \text { دورت } \\
& \text { لj elli cinquante } \\
& \text { bech cinq } \\
& \text { alty six } \\
& \text { يلى iedi sept } \\
& \text { سكز } \\
& \text { dhokouz neuf } \\
& \text { نी on dix } \\
& \text { آلتشنش altmych soixante } \\
& \text { ietmich soixante-dix } \\
& \text { سكسان seksan quatre-ringt } \\
& \text { dhogsan quatre-vingt-dix } \\
& \text { iouz cent } \\
& \text { bin mille } \\
& \text { يوزبيك } \\
& \text { iiguirmi vingt } \\
& \text { مليبون milioun million }
\end{aligned}
$$

2. Les noms de nombre cardinaux composés se forment par juxtaposition, p.e. أونأيكى douze, يوز سعسان دورت cent quatrevingt-quatre, أوتوز 10533 etc. Le nombre le plus grand se met toujours le premier.

3. Les noms de nombre ordinaux se forment des noms de nombre cardinaux en ajoutant à la fin ndji ou indji, p. e. برنحجى birindji

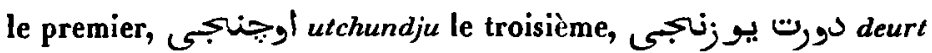

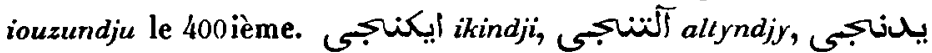

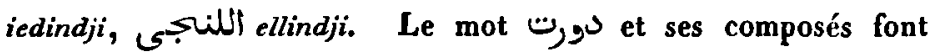
 


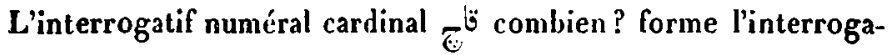
tif numéral ordinal ثنجئي qatchyndjy le combien?

4. Pour indiquer la distribution relative on ajoute un, aux noms de nombres cardinaux turcs, p.e. بر lirer un à chacun, أوجر trois à chacun. Mais si la dernière lettre du nombre cardinal est une voyelle on intercale un $\dot{ش}$ entre celle-ci et le r, p. e. أيكيشتر ikicher deux à chacun, آلتيثي altychar six à chacun.

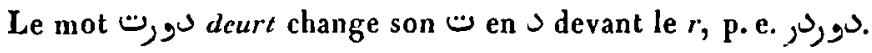

Pour les centaines et les milliers, c'est le mot qui en indique le nombre qui prend le signe du distributif, p. e. ألنينثر بيكث altychar biñ sept mille à chacun.

5. Les fractions sont généralement formées par deux noms de nombre cardinaux turcs: celui de deux qui indique le dénominateur de la fraction se place le paremier et prend la postposition s.: après lui. Le nombre qui suit est le numérateur: ايكبيده بـ ikidé bir un sur deux, أونله أولر trois sur dix. On ajoute assez souvent l'un

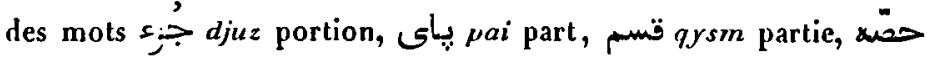
nysca lot, ì chaque partie de la proposition fractionnaire, p. e.

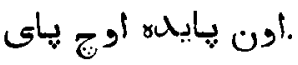

On emploie aussi les nombres fractionnaires arabes jusqu'aux dixièmes pour les dénominateurs et les noms de nombre cardinaux turcs pour les numérateurs: بر نصف bir nisf une moitié, ورب utch rub' trois quarts etc. Les numératifs fractionnaires depuis un

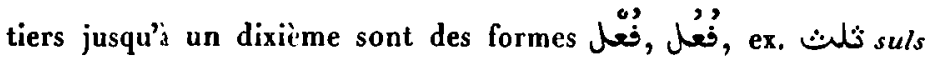
et sulus, سلس suds et sudus un sixième, ثمل sumn et sumun un huitième.

Voici les formes des chiffres que l'on emploie en arabe, persan et turc:

$$
\begin{array}{llllllllll}
1 & \kappa & \mu & 5 & 0 & 4 & \vee & \wedge & 9 & . \\
1 & 2 & 3 & 4 & 5 & 6 & 7 & 8 & 9 & 0
\end{array}
$$




\section{XVIII}

\section{LE PRONOM.}

1. La classe des pronoms comprend les pronoms personnels, les pronoms démonstratifs et les pronoms interrogatifs.

Table des pronoms personnels.

\section{Siugulier.}
1. prs.
2. prs.
3. prs.

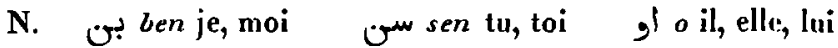

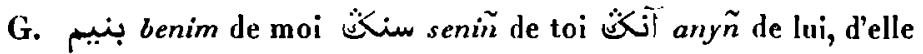

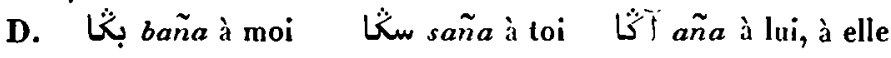

A. بنى beni moi, me سنى seni toi, te بny le, la

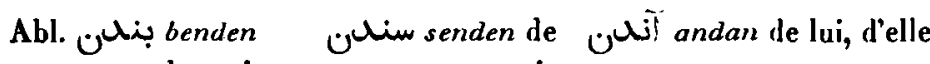
de noi

toi

\section{Pluriel.}

N. بز biz nous آنَل

G. بزم " bizim de nous "

2. Le pronom réfléchi est représenté dans la langue ottomane par le mot كنب كنl kendi. Singulier.
Pluriel.
1.prs. كنl kendim
كن ken- moi-même dimiz nous - mêmes
2.prs. كنل كن , kendiñ كن kنl- diniz vous-mêmes toi - même

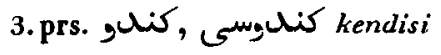 lui -, elle-même كil ken- diler eux-, elles - mêmes

3. Les pronoms démonstratifs sont en turc: أننبو ou bou ceci, celui-ci, celle-ci; نشو chou ou gl o celui - là, celle - là. En persan :

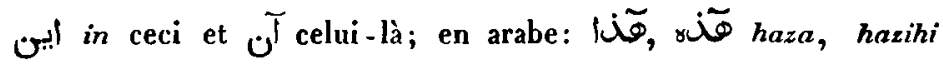
ceci et $u \sqrt{\bar{j}}$ zalikr celui - li. 


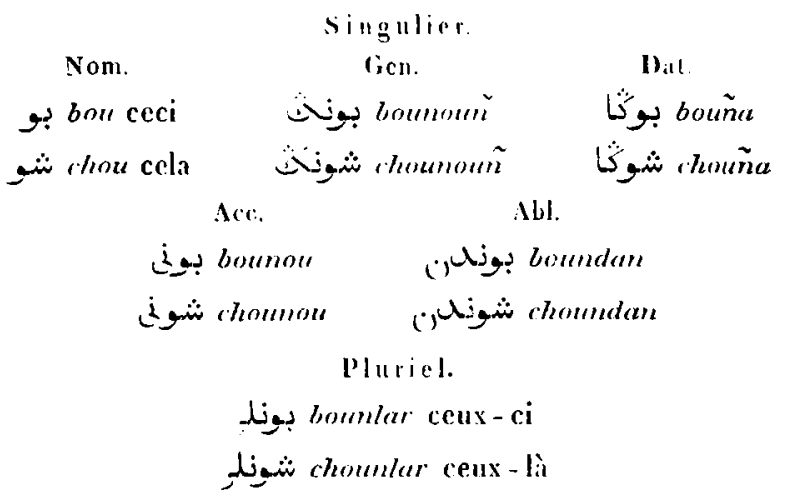

1. Les pronoms interrogatif's sont: كيب kim qui? pour les

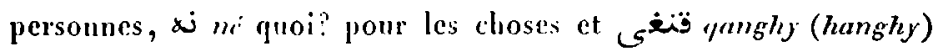
lequel, lanuelle? pour les personnes et les choses ígalement. Le

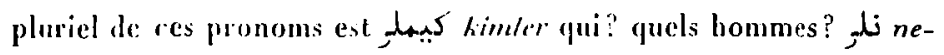

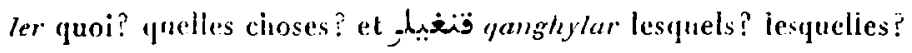

Ces trois mots et les autres interrogatifs simples ou composés,

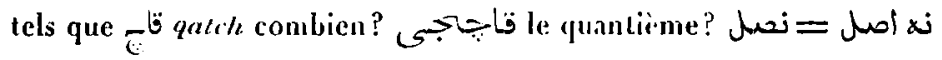

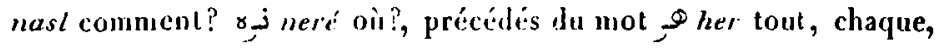

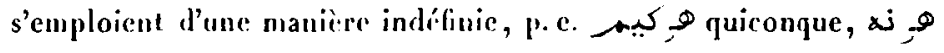

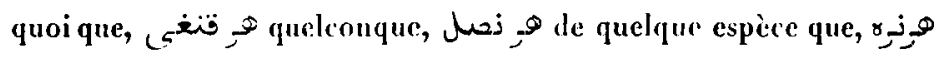
quelque entroit que.

5. Le seul affixe pronominal relatif est 6 , ki. On s'en sert pour les personnes et pour les choses; il est tour à tour adjectif et substantif. Lin qualité d'affise il s'attache aux noms et aux pronoms

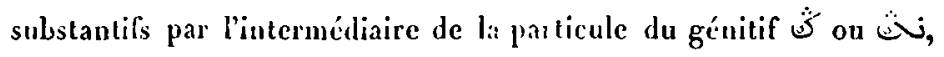
p. e.:

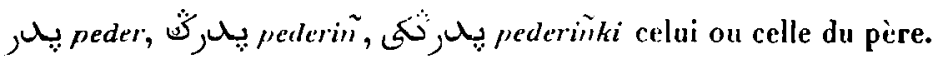
w sen,

بو bon, boumouniti celui qui est ì lui, le sien. بن benimki celui qui m'appartient, le mien.

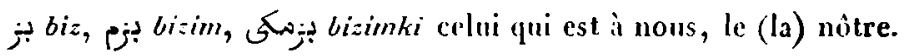




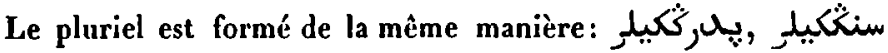
seninkiler.

L'affixe pronominal possessif introduit dans cette combinaison se place immédiatement après le nom:

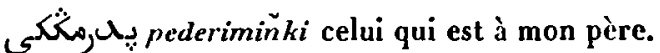

Ce même affixe peut ètre ajouté à un nom au locatif:

, بِ celui qui est auprès du père,

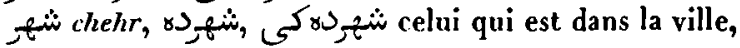

بنlui qui est auprès de moi.

Cet affixe كى se combine aussi sans l'intervention d'aucune préposition avec les noms qui font la fonction d'adverbes de temps:

بujourd'hui, بوكونك bougiounki celui d'aujourd'hui, نون دونك dunki celui d'hier,

صباح sabahki celui du matin.

\section{E R B E.}

R e m a r que s.

1. Par les exemples donnés dans la table ci-jointe il sera évident que les verbes sont composés d'une racine immuable et d'un nombre de terminaisons.

2. On forme les dérivés d'un verbe en ajoutant à la racine différentes syllabes, savoir: in pour le réfléchi, ich pour le réciproque, dir pour le causatif, ma, me $\left(\rho, \omega, x_{0}\right)$ *) pour le négatif, ama, eme (wr, xor) pour l'impossible, il pour le passif. Le grand nombre de verbes dérivés résulte de la combinaison de plusieurs syllabes de cette espìce.

3. L'idée représentée par la plupart des dérivés du verbe est fort évidente, elle est déjà expliquée par leurs dénominations: le réciproque en ich signifie faire mutuellement, le causatif en dir lais-

*) Les lettres l et $\checkmark$ dans le négatif sont des lettres de direction et sont généralement omises tandis que le $\gamma$ de l'impossible est un signe caractéristique. 
ser faire, le négatif en $m a, m e ́$ ne pas faire, l'impossible en ama, emé ne pouvoir faire et le passif en it itre l'objot de l'action. Ce n'est que la valeur du verbe rétléchi qui offre quelque difficulté; car bien qu'elle ait quelquefois un objet direct, l'action de ce verbe ne

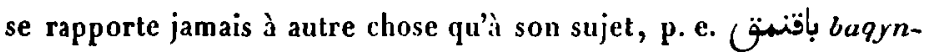
maq regarder çà et là sans objet fixe, كنمكن guezinmek se promener seulement pour l'exercice.

i. Le passif des verbes neutres, rélléchis et réciproques a quelque chose d'étranger pour nuus. Ces formes n'énoncent pas une action passive directe du sujet, mais l'idée exprimée par ces formes est: être ou pouvoir ètre le moyen par lequel, on l'objet pour lequel l'action exprimée par la forme active se fait, p. e. كتمك guilmek, كيدلك guidilmek *) ètre tel qu'on puisse y aller ou qu'on puisse aller par- là.

5. Le causatif est formé génćralement par 0 dir, mais si la dernì̀re syllabe d'une racine se compose de deux consonnes avec ou saus lettre de direction ou de prolongation entre elles el dont la derniìre est $\mathcal{J}$ ou, : alors le causatif se forme en ajoutant un quiescent au lieu de la syllabe dir, p. e. كتور guetirmek, caus. guetirtmek faire apporter, كتورتمكن

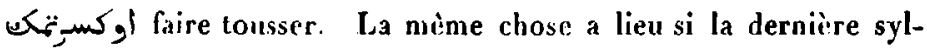
labe est composée d'une seule consonne avec sa voyelle, p.e. اوتوني lire, أوقوتمخ oqoutmaq faire lire. Il y a encore beaucoup de verbes qui subissent différentes irrégularilés en formant le causatif. Souvent, par exemple, on supprime le $d$ en conservant le $r$ seul:

*) Le ت se change en $\lrcorner$, cmp. la règle donnée ci-dessus p. III, 5 .

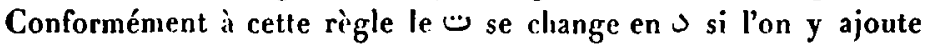
une syllabe commençant par une voyelle, cf. إيـانمسك idinmek, إيلشهك idichmek faire réciproquement, إبلاهمك idemémek ne pas pouvoir faire. 


\section{XXII}

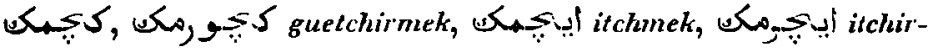
mek boire.

6. Les racines dont la derniìre lettre radicale est une $J$ et celles qui finissent par une voyelle, forment leur passif en ajoutant à la racine un $\mathcal{U}$ an lieu de $\mathcal{J}$. Le passif de ces verbes devient donc identique pour la forme avec le rófléchi, cf. pris, بولنمهن boulounmaq ètre trouvé,

7. Si la derniere consonne d'une racine est munie d'une voyelle on intercale dans les verbes impossibles la syllabe بa au lieu de

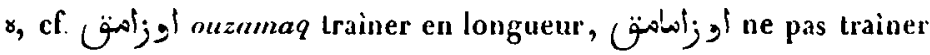

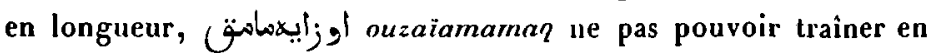
longueur.

8. On peut ajouter aux verbes dérivés une espìce de verbes résultant du rapprochement de deux verbes dont le deuxième se conjugue tandis que da premier il ne reste que la racine terminée en $e$ ou en ïe accessoires. C'est au moyen de cette périphrase qu'on exprime la possibilití si le deuxième verbe est بلمه bilmek (savoir, pouvoir), ou la facilité s'il est بيريكن virmek donner.

\section{PARADIGME DU VERBE.}

Nous donnerons les paradigmes d'une racine forte et d'une racine faible, بلمَك brendre et bilmek savoir.

\section{Impératif.}
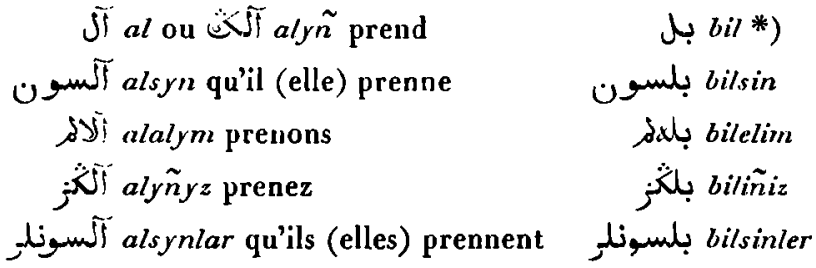

*) La seconde personne de l'impératif est représentíe par la racine seule. 
Présent difini.

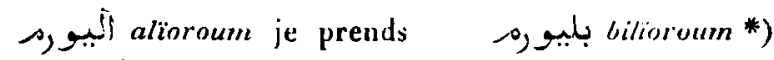

$$
\begin{aligned}
& \text { (ألبيو رسن aliorsoun } \\
& \text { أليو اليبو } \\
& \text { aliorsouñouz } \\
& \text { آلبيو رليور }
\end{aligned}
$$$$
\text { (dans cet instant mème) }
$$

$$
\text { biliorsoun }
$$$$
\text { bilior }
$$$$
\text { بليبو biliorouz }
$$$$
\text { biliorsounouz }
$$$$
\text { (iliorlar }
$$

Imparfait détini.

al or idim je prenais (dans cet instant mème)

آليو, البيد البه

bilï̄r idirm etc.

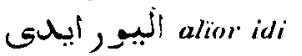

بلبيور أبيلث

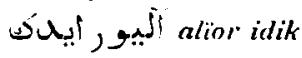

بليو ر أيلى ايليو

alior idiniz

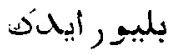

أنبيور أيليلز

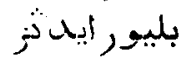

بليبو رابيليله:

$$
\text { Présent indéfiui (Aoriste)**) }
$$

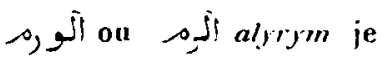
prends (habituellement)

et bilirim. je prendrai

ou li alyrsyn

bilirsin"

آل

j, il ou ; alyryz

bilir

alyrsyriz

biliriz

Jit ou

بانِ bilirsiniz

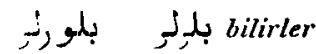

*) Cf. pag. VII, 10. Le présent défini est très déterminé dans son rapport au présent.

**) Le présent indéfini indique plutòt une habitude générale, il se rapporte aussi au futur et implique alors une chose qui doit se faire encore. Cette forme est employće très souvent comme aoriste dans les narrations. La voyelle qui précède le $r$ de l'aoriste est un 


\section{XXIV}

\section{Passé.}

alyr idim ou أنو ألمور أيله alyrdym je prenais (habituellement),

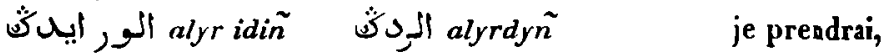
alyridi الريى alyrdy j'aurais pris

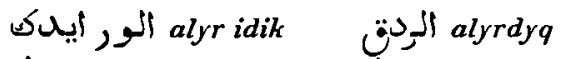

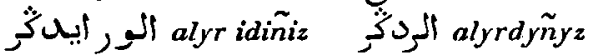
alyr idiler الربلر الريلر البيليلر

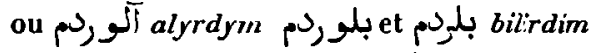

$$
\begin{aligned}
& \text { bilirdiñ } \\
& \text { bilirdi } \\
& \text { bilirdik } \\
& \text { bilirdiniz } \\
& \text { Parfait. } \\
& \text { bilirdiler }
\end{aligned}
$$

sull aldym j'ai pris بلدildim

ثิ aldyn نildiñ

Guit aldy vildi

ơl aldyq bildik

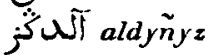
بلن sildiniz aldylar بلبيلر Plusqueparfait.

في آلّ بلايله bididim

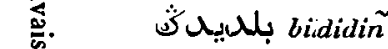
bididi biididik biltidiniz biltidiler $a$ ou un $i$, qui selon la loi de l'euphonie se prononcent différem-

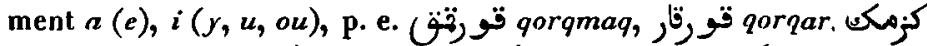

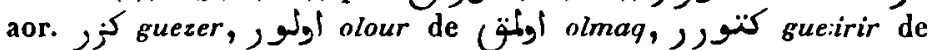
. Pour indiquer les voyelles on se sert souvent d'un l pour a el d'un, pour $i, y, u$, ou; mais le l et le g n'étant que des lettres de direction on les omet aussi souvent. 
Parfait inditerminé.

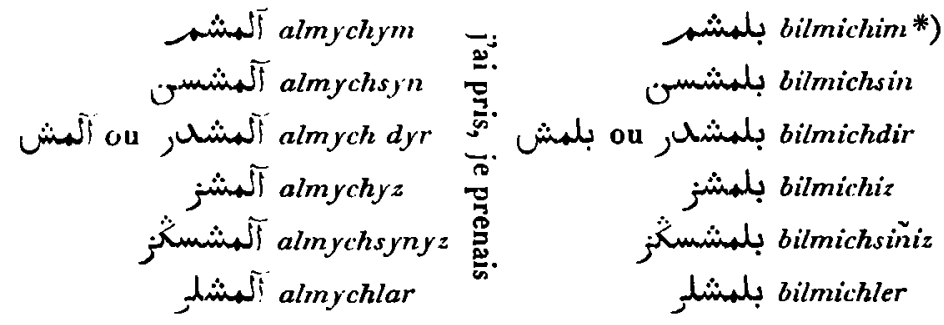

Plusqueparfait indéterminć.

آلمشند almychdym j'avais pris

آلمنشيثن almychdyn

Bilmichdim

آلمشن almychdy

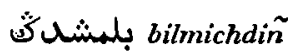

آلمشنق almychdyq بالمشندى bilmichdi

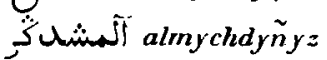
bilmichdik

آلمشيديل بلفيشن bilmichdiniz bilmichdiler

Futur.

aladjaghym je vais prendre

أله aladjaqsyn

ق ألغجن

أل aladjaghyz

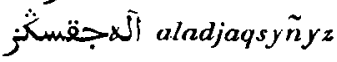

aladjaqlar

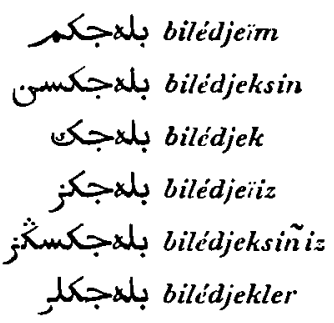

Futur passé.

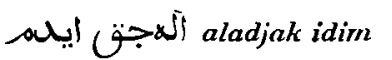

biledjekidim etc.

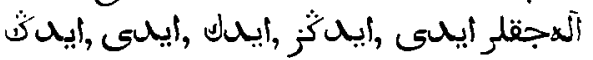

aladjaglaridi

*) Ce parfait se compose du participe parfait en مش et du verbe

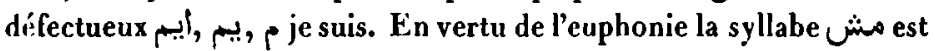
prononcée différenment, ex. أولشن ; اوروهت eulmuch de أولمك 


\section{XXVI}

\section{Nécessitatif.}

almalyiym il faut que je almalysyn prenne

almaly

bilmel im

almalyijz

آلهلوسگز almalysyñzz

آلملول

bilmelisin

bilmeli

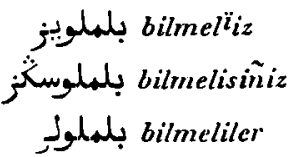

Parfait.

almaly idim il me

bilmeli idim

fallait prendre

Optatif.

Présent.

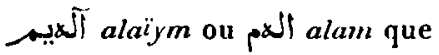

نumasyn je premne

íla ala

بiléiim

نilisin

بilé

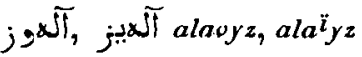

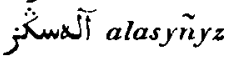

oiléviz, bileiiiz

Jallar

bilesiñiz

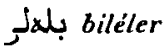

Parfait.

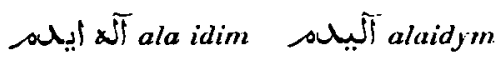

(يلم إيلم

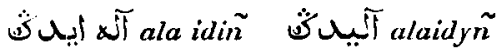

bileïdim بليحه

آلّ آليدى ala idi alaidy

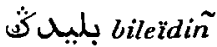

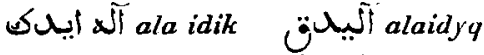

bileidi

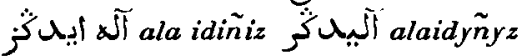

bileidik

alaidylar

\section{Conditionnel (Aoriste.)}

آلس alsam si je prends

isañ

bilsem بلسmهر

بلسيكت 
and alsa

(t)ing alsan

iti alsariyz

inni alsalar lilse"

بلسيك

بلمئر bilseniz

Lilseler

\section{Parlait.}

alisaridym si je pris, beilseilim

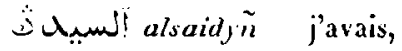

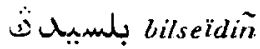

Eيtris alsaidy j'eusse pris bilseidi

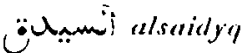
bilseidik

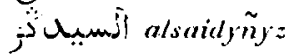
بإسبيدن bilseidiniz

alsaidylar.

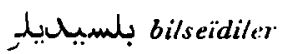

\section{Infinitif.}

almaq prendre

بilmek savoir

Participe.

Prísent (j) alan qui prend

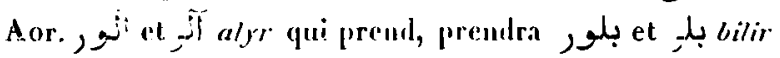

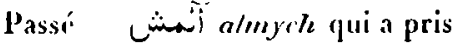
bilmich بلمنشن بلز

Parfait ¿Uil aldyy qui a pris (بله cildik

Futur ( ن بانdédjek

Noms verbaux.

Présent alma (l'action d')ouvrir

Parfait ölit aldyq (l'action d')avoir ouvert

Futur $(j$ all aladjay (l'action de) devoir ouvrir

cilmé (l'action de) savoir *)

بuldik

بل liledjek

*) Les noms verbaux sont déclinés tout - à - fait comme les noms,

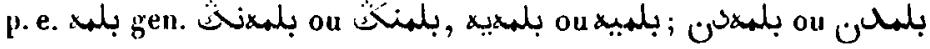
in omettant ou mettant le x. 
Gérondifs.

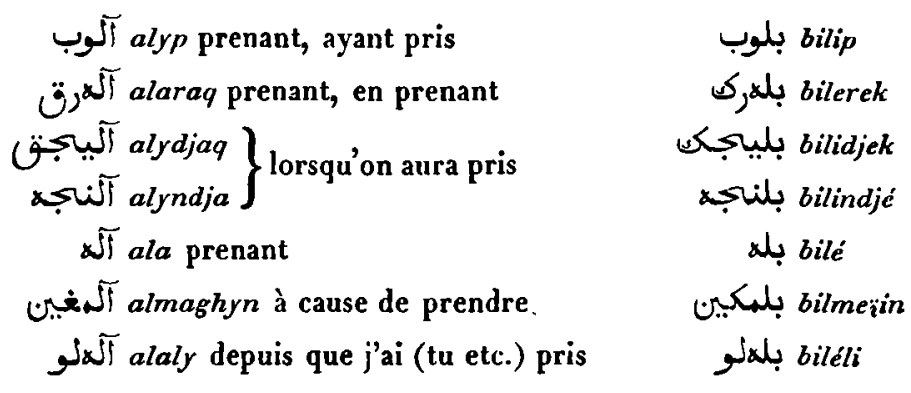

Modèle

de l'Aoriste de l'Indicatif d'un verbe négatif.

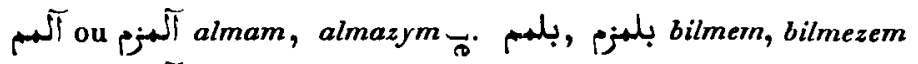

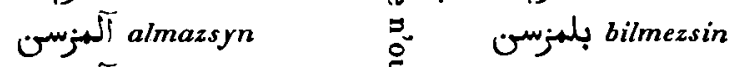

آلمز almaz بلم bilmez

آلمبز oulmaïyz, almazyz

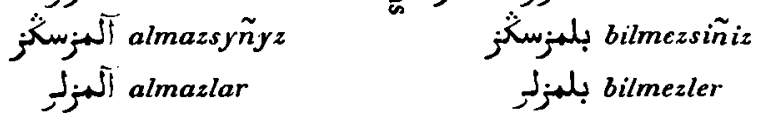

Modèle

de l'Aoriste de l'Indicatif d'un verbe impossible.

مoxل alamarn, آلكز alamazym je ne puis, pourrais pas prendre

alamazsyn

زoلli alamaz

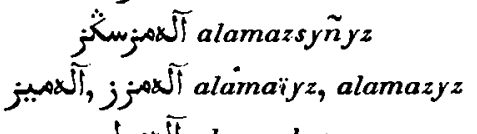

alumazlar

bilcmem, بلماهز bilemezim

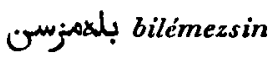

بilémez

bilémeiz, bilemeziz

بلمهنزئز bilemezsiniz

بلهزئر bilémezler 


\section{XXIX}

Chacun des verbes simples dérivís a une conjugaison entière qui est conforme en toutes choses au modele que nous venons donner.

Nous indiquons ici la conjugaison d'un verbe passif.

P a s s if.

\section{Impératif.}

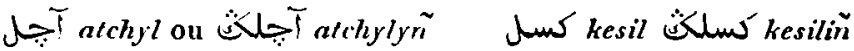

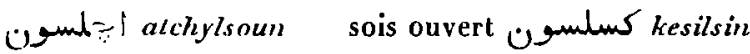

آججلئم atchylalym

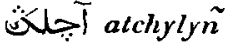

كسلهلم Kesililim

(i) atchylsoun كسلكن Kesiliñ

Tَج atchylsounlar كسلسونلى

Présent.

كesilioroum

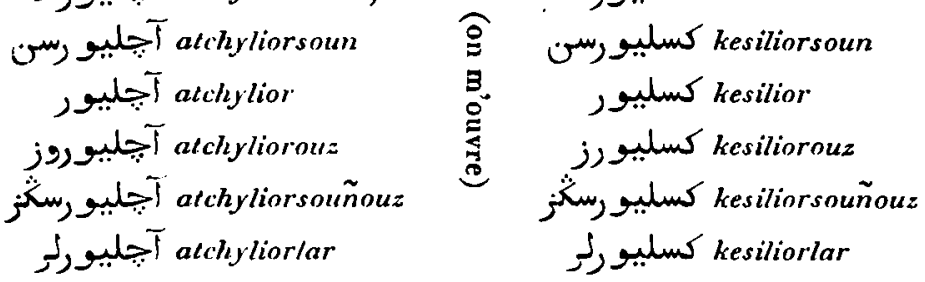

Imparfait.

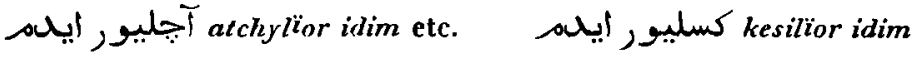

Aoriste.

آجخلور atchylyrym je suis

كسلو كesilirim Kesilirsin

atchylyr

كسلور كسلور

j) atchylyryz

atchylyrsyñzz

كesiliriz

atchylyrlar

كسلو رسكز; كesilirsiniz

كسلورلر كesilirler

P assé.

atchylyr idim

كesilir idim 


\section{Parfait.}

oul atchyldym j'ai été ou-

كسلم kesildim

آَجْلد atchyldyñ

vert كسلى kesildin"

أجبلى atchyldy

كسيh kesildi

آجَجِ atchyldy

Kesildik

آَجلن atchyldyñzz

كسلف كسلز

آجيلبيل آجليل aichyldylar

Plusqueparfait.

atchyldym idi

Kesildim idi

Futur.

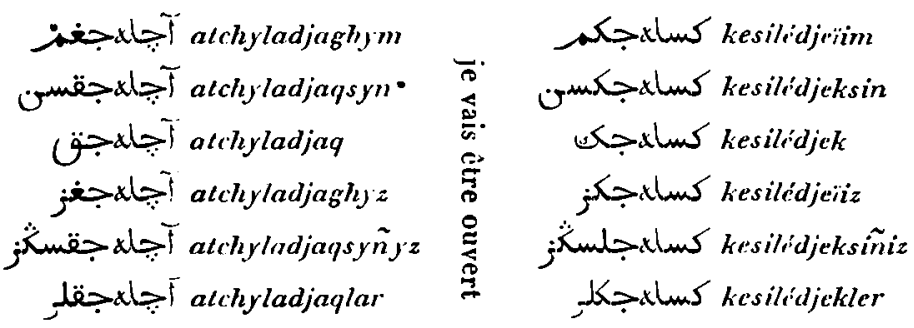

Futur passí.

كesiledjek idim

Nécessitatif. Présent.
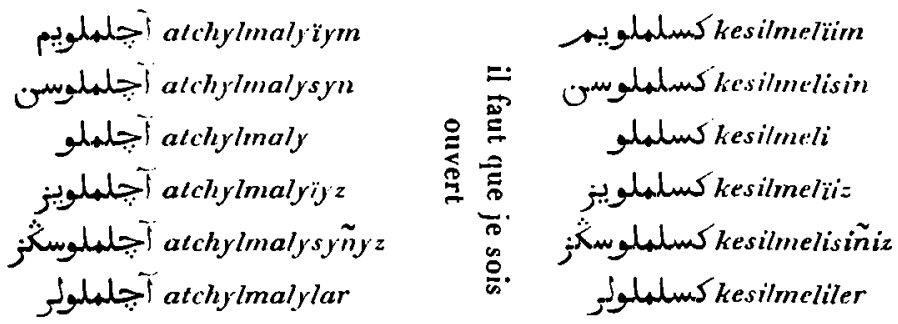

Parfait.

atchylmaly idim

كسيملو أيله kesilmeli idim 


\section{XXXI}

Optatif. Présent.

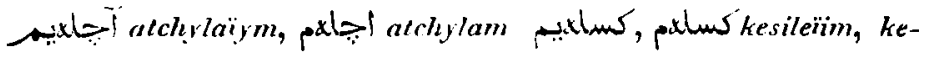
que je sois ouvert silem

$$
\begin{aligned}
& \text { ن } \\
& \text { أبا atchyla }
\end{aligned}
$$

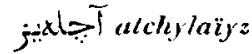

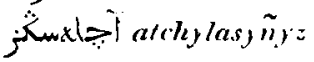

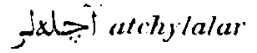

كسidesilesin Sulles

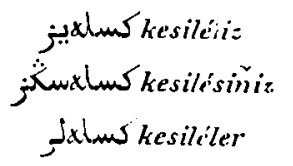

Parfait.

atchylä̈lym que je كسلبيد kesileïdim

fusse ouvert

$$
\begin{aligned}
& \text { } \\
& \text { آججليدى atchylaïdy }
\end{aligned}
$$

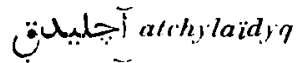

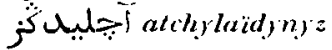

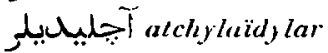

$$
\begin{aligned}
& \text { كسليدكثن } \\
& \text { كسلبيدى كesileidi } \\
& \text { كسليد: كesileìdik }
\end{aligned}
$$

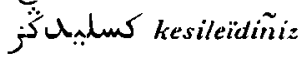

$$
\begin{aligned}
& \text { resileidiler. }
\end{aligned}
$$

Conditionnel. Aoriste.

آَجلسم , آججلسكايم alchylsam si je suis ouvert

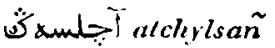

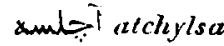

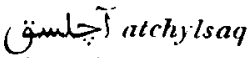

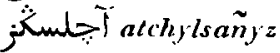

$$
\begin{aligned}
& \text { atchylsalar. }
\end{aligned}
$$

كسلmasilsem

$$
\begin{aligned}
& \text { كسلسكت Kesils"ñ } \\
& \text { كndmesilsé } \\
& \text { كسلسك } \\
& \text { كسلسفخز Kesilseñiz } \\
& \text { كسلسגل }
\end{aligned}
$$

\section{Parfait.}

آجّلسيبه alchylsaïdym si j'é-

tais, avais été, cusse été ouvert

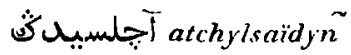
Talchylsaidy
Kesilseïdim كسليسيل Kesilseidi 


\section{XXXII}

$$
\begin{aligned}
& \text { atchylsaidyg }
\end{aligned}
$$

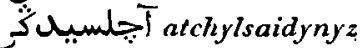

$$
\begin{aligned}
& \text { atchylsaidylar }
\end{aligned}
$$$$
\text { Kesilseidik }
$$

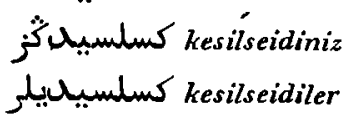

\section{Infinitif.}

آجيجلمق atchylmaq être ouvert

كسلمكن kesilmek

$$
\text { Participes actifs. }
$$

Présent آنجلان atchylan qui est ouvert

كسلان kesilen

Aoriste آَجلور acchylyr qui est, sera ouvert

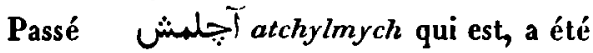
ouvert

$$
\text { كسلور }
$$

كسلهنش kesilmich

Parfait $\ddot{ن} M \backslash T$ T atchyldyq qui est, a été ouvert كسلטك kesildik

Futur آَجلهجن atchyladjaq qui va être ouvert

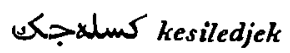

Participes passifs.

Aoriste à, de qui on est ouvert كسلגك kesildik

Futur آَجلهج atchyladjaq par, avec, dans, à, de qui on va être ouvert كسلغجكن kesilédjek

Noms verbaux.

Présent آنجلي atchylma être ouvert كسبلهי Kesilmé

Parfait آَجللن atchyldyq avoir été ouvert

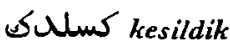

Futur آجَلهجن atchyladjaq devoir être ouvert | kesilédjek

Gérondifs.

atchylyb ayant été ouvert kesilib

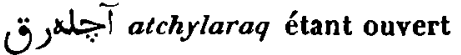
كسلفرك (آجليبجن atchylydjaq) quand je etc., étais,

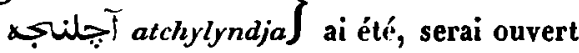


XXXIII

Totchyla étant ouvert

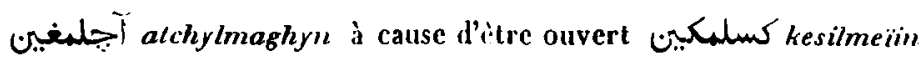

iَ alchylaly depuis que je etc. suis,

étais, ai été ouvert

$$
\text { Verbes a uxiliaires. }
$$

Parmi les verbes simples il y en a qui servent d'auxilaires. Les verbes auxiliaires qui sont d'un usage très fréquent sont: ايتمكك

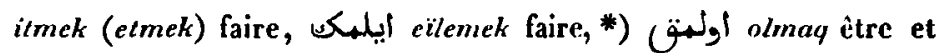
devenir, $\hat{\vartheta}^{\prime}$ lolounmaq être fait, devenir. Ces verbes se conjuguent d'aprìs les modèles donnés ci-dessus et c'est pour cela que nous ne donnons ici que la troisième personne du singulier de chacun de leurs temps.

Impératif

\section{Indicatif.}

Présent

$$
\text { أيبت it, et fais }
$$

alle:

Imparfait

إيليبيور idior il fait

أيليور

\section{faisait}

$$
\text { إيلبيور أبلى idior idi il }
$$

Aoriste

$$
\text { إيبر ider il fait, fera }
$$

$$
\text { eileïor idi }
$$

$$
\text { Passé إبيلر إبلى ider idi il faisait, }
$$

Parfait إيتلى أتلى ildi il fit,

$$
\text { il a fait إيلانى eilledi }
$$

Plusqueparfait إيتل يجلى itdidi, etti-idi il

$$
\text { avait fait إيلل يبلى eiledidi }
$$

Futur إبلهجكى idédjek il va

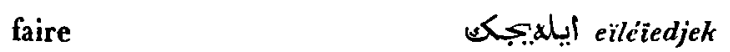

*) Le verbe signification, mais cela arrive moins souvent dans la langue ottomane que dans le dialecte du Tchagataï. 


\section{XXXIV}

Futur passé أيلهجك أيلى idédjek idi il

allait faire

ज أبلإsileiedjek idi

Nécessitatif.

Présent إيتهلو itmeli il faut qu'il fasse إيلهلو

Parfait إيتهلو أيخى elmeli idi il fallait

qu'il fut

أيللو أيلى eilimeli idi

Optatif.

Présent إيلها idé qu'il fasse eileäé

Parfait أيله أيلى idé idi qu'il fit, qu'il eǹt fait إيله أيلى eile idi

Conditionnel.

Aoriste إبتس ilsé s'il fait eilesé

Parfait أيتسبيلى itseidi s'il faisait, avait fait إيلسبيدى eilesez̈di

Infinitif إبيلمكك itmek (etmek) faire إيتهك illemek

Participes actifs.

Présent أبيان iden qui fait

Aoriste إيبر ider qui fait, fera

Passé أيتهش itmich qui a fait

eilen

Parfait أيتشك أينشك itdik qui a fait

إيلر eiller

Futur أيبكان idédjek qui va faire

eilemich

أيلاك eiledik

إيلهيكيكن eiléiedjek

Participes passifs.

Aoriste إيتلك itdik qui est fait (par un agent qui fait)

أيللك elledik

Futur ايلهجك idédjck qui va être fait

إيلهبs eillëiedjek

Noms verbaux.

Présent إيتمه itmé (l'action de) faire

أيلمن eilemé

Parfait أيتلك itdik (l'action d')avoir fait

أيلدك eïledik

Futur أبلاهجك idédjek (l'action de) devoir

faire

إيلهيجمك eilëiedjek 
Gérondifs.

idib faisant, ayant fail إيلبوب cilciil

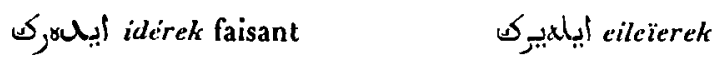

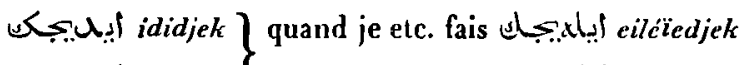

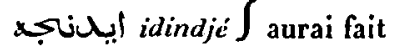

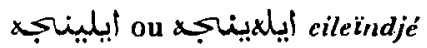

ช إئlé faisant

أيله eilé

أيتمكين ilmeïn à cause de faire

نيكان cilemeiin

أبيلو ideli puisque je etc. fais

إيانيلو ايليكي

Exemples de ces verbes auxiliaires :

تقنسبم أيتلى , أيلدى taqsin itdi eiledi il a fait la division, il a divisé;

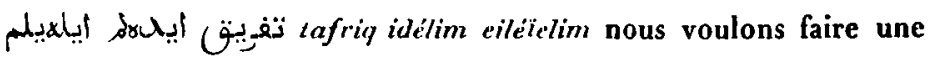
séparation, séparons.

Conjugaison du verbe auxiliaire $($ lolmaq être et de son Passif (لنمتز; olounmaq.

Indicat if.

Impératif

Jol ol sois

نololinun

Prísent $\quad$ olior il est

, olomion.

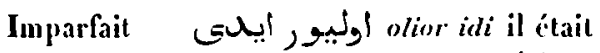

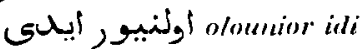

Aoriste

ر اوليو

Passí

, flounour

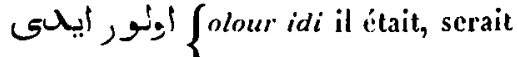

जo hourdou il aurait ití

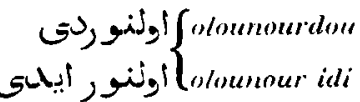

Parfait

sujgl oldou il fut, a cití

suij, sloundou 


\section{XXXVI}

Plusqueparf.

$$
\text { जيligloloundoudou }
$$

أولنبى أبلنى oloundou idi

Futur $\quad$ ( $\quad$ (

$$
\text { (أن أنهاج olounadjaq }
$$

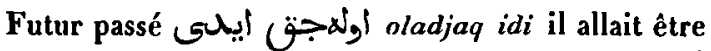

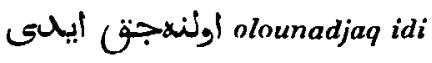

\section{Nécessitatif.}

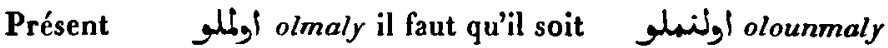

Parfait أولملو أيلى olmaly idi il fallait qu'il fût, il devait, aurait du être أنسلو أيلى olounnaly idi

$$
\text { Optatif. }
$$

Présent لýl ola qu'il soit

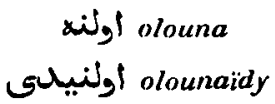

Parfait أوليبلى olaïdy qu'il fùt, eût été

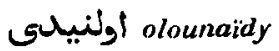

Conditionuel.

Aoriste $\quad x$ glol olsa s'il est ligunsa

Parfait eût été

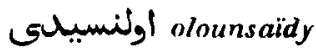

\section{Infinitif.}

Présent أولمق olmıa

أولنمتج olounmaq

Participes actifs.

Présent $\quad ن^{ل / a l a n ~ q u i ~ e s t ~}$

Aoriste اولور أولور olour qui est, sera

Passé $\quad$ injolmouch qui a été

Parfait ölyl oldouq qui a été

Futur

$$
\begin{aligned}
& \text { اوننور } \\
& \text { ش ألنشه olounmouch. } \\
& \text { أولنبان oloundoun }
\end{aligned}
$$

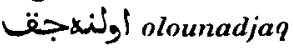

Participes passifs.

Aoriste قUلigloloundoun 
Futur أولعجق oladjaq par, avec, à, de, dans qui on va ètre

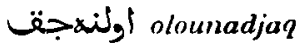

Noms verbaux.

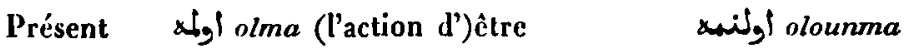

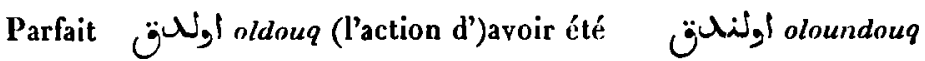
Futur (الزلججن oladjaq (l'action de) devoir ètre

جن lounadjaq

Gérondifs.

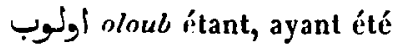

olounoub

اولثر olaraq étant, en étant

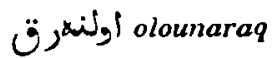

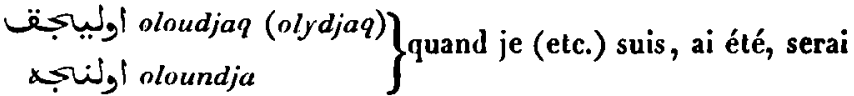

olounydjaq

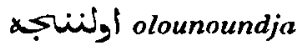

Nol ola étant

أولنغ أولني

أو olmaghyn à cause d'ètre

iflounmaghyn

أولى olaly depuis que je (etc.) suis, ai été

olounaly

On forme par moyen de ces verbes une grande quantité de verbes composés, p. e.:

zahyr olmaq être évident, se manifester

qath' olounour il est, sera coupé

zikr olounour il est, sera mentionné.

Du verbe substantif.

Il y a deux autres verbes substantifs qui sont très souvent employés comme une espèce d'auxiliaires. Ces deux verbes sont défectueux. Le premier en est $د$ dir c'est, il est, au pluriel $د$ dir. ler. Il sert d'affirmation on de liaison logique entre le sujet et son attribut. Le deuxième de ces verbes: يمر (يمر (م) se conjugue de la manière suivante: 


\section{XXXVIII}

\section{Impératif manque.}

\section{Indic at if.}

Présent.

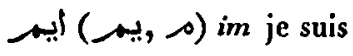

sin tu es

III. prs. manque

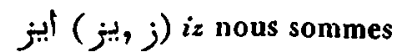

سخغز siniz vous êtes

III. prs. manque

Parfait.

as, al dim, idim j'étais, je إيلى dik, idik

fus, j’ai été

گे), din, idin

نيخ diniz, idiniz

ज), أيلى di, idi

diler, idiler

Nécessitatif et Optatif

manquent.

Conditionnel.

Aoriste.

Parfait.

أبيسم isem si je suis

إيسيس أيسن iseñ

أبسx isé

إيسكك isek

أيسخر iseñiz

أيسيسكر إيسر iséler

أبسيله iseïdim si j'ćlais, fus, إبيسبيدثي iseidin

أبسبيليى iseidi

$\cong$.

إبسيبك iseidik

أبسيبلن iseiddiniz

iseidiler

\section{Infinitif et Participes}

manquent.

Noms verbaux.

\section{Présent manque}

Parfait إيدك idik (l'action de déjà) être

Futur manque

Gérondif.

إيكى étant.

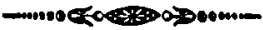


TEXTES. 
